\title{
CB6F1-TgN (RasH2)
}

National Cancer Institute

\section{Source}

National Cancer Institute. CB6F1-TgN (RasH2). NCl Thesaurus. Code C98711.

A transgenic mouse at F1 generation with background strain C57BL/6 crossed with

BALB/CAn, containing three copies of the human c-Ha-Ras gene introduced in tandem. 